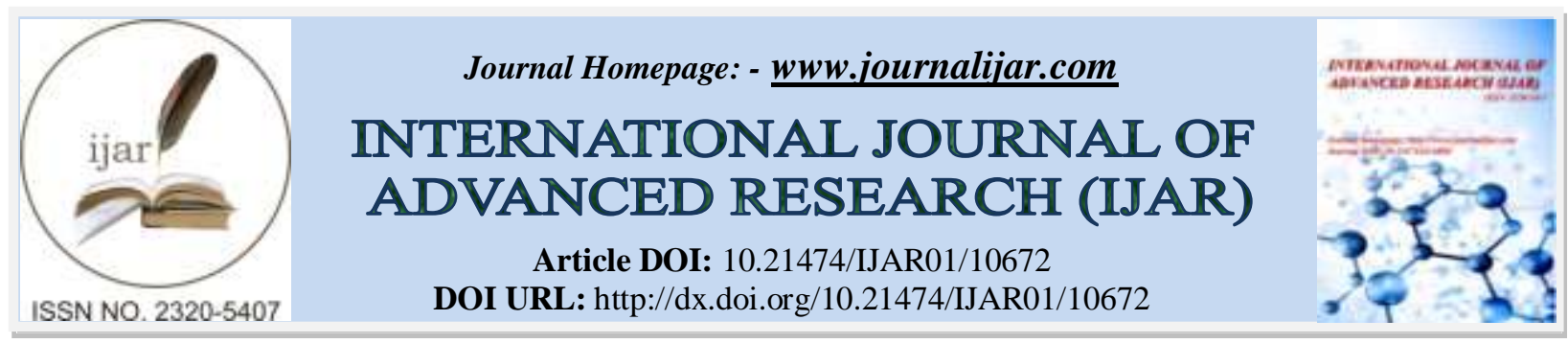

RESEARCH ARTICLE

\title{
ISOLATION AND IDENTIFICATION OF ENTEROCOCCUS SPP. COLLECTED FROM CENTRAL REFERRAL HOSPITAL, MANIPAL FOR CLINICAL SAMPLES AS WELL AS ENVIRONMENTAL SAMPLE COLLECTED FROM GANGTOK REGION
}

\author{
Prasanta Protim Hazarika ${ }^{1}$, Tashmi Konwar ${ }^{2}$ and Amrit Kumar Hazarika ${ }^{2}$ \\ 1. Sikkim University, School of Life Science, Microbiology, Gangtok, 737102, Sikkim. \\ 2. Sikkim University, School of Life Science, Botany, Gangtok, 737102, Sikkim.
}

\section{Manuscript Info}

Manuscript History

Received: 15 January 2020

Final Accepted: 17 February 2020

Published: March 2020

Key words:-

Enterococci, Vancomycin, VRE, MIC

\begin{abstract}
The emergence of Vancomycin registant enterococcus (VRE) has become a major problem particularly among immunocompromisied patients. In the normal healthy host enterococci seldom are causes urinary tract infections, abdominal infections followed by bacteremia, endocarditis and meningitis rarely. The treatment of enterococcal affliction has become more challenging due to increasing resistance against VRE strain. The entire collected 50 samples were identified by gram staining, MacConkey agar, Bile esculin agar, M- Enterococcus agar, blood agar and among all of them Enterococcus spp. was found to become the predominant species in the studied region. To speculate the antibiotic susceptibility pattern for vancomycin, the minimum inhibitory test (MIC) was done for enterococcal species which having the resistivity against vancomycin was $22.8 \%$. This finding may indicates that increasing resistivity associated with significant increase in consumption of greater amount of antibiotics for severe bacterial infection. The increase in resistance patterns reflects that limited treatment options might create a risk in future prospects for managing complicated cases.
\end{abstract}

Copy Right, IJAR, 2020,. All rights reserved.

\section{Introduction:-}

The emergence of enterococci is now becoming an important etiogen in nosocomial and community acquired infections. It is an opportunistic pathogen which can cause severe infection in human, particularly among immunocompromisied patients (Zirakzadeh and Patel, 2006). The enterococci are gram-positive, facultatively anaerobic bacteria which can able to form a chain of various lengths; and can survive under harsh conditions (including high salt concentrations) and also at a wide range of temperatures (from $10{ }^{\circ} \mathrm{C}$ to $>45{ }^{\circ} \mathrm{C}$ ). Enterococci are also intrinsically resistant to a broad spectrum of antimicrobial agents and as it increased in prevalence worldwide as pathogens in nosocomial infections, antimicrobials have been used in greater frequency in hospitals (Kak and Chow, 2002). Vancomycin resistant Enterococci (VRE), reported initially in Europe in 1988, raised a threat to public health. Mutters et al. (2013) reported that VRE bacteraemia was found exclusively in multimorbid patients. Enterococci with acquired vancomycin resistance are a major concern in the hospitals. Their importance has also increased due to the spread of its resistance to Staphylococcus. This resistant species has the ability to survive for long periods and can spread to patients from health care workers.

Corresponding Author:- Prasanta Protim Hazarika

Address:- Sikkim University, School of Life Science, Microbiology, Gangtok, 737102, Sikkim. 
In the colon of nearly all humans, enterococci are found in numbers as high as 108 colony-forming units per gram of feces (Moore and Holdeman, 1974). In humans, vancomycin-resistant enterococci (VRE) most commonly result in intestinal colonization, which could endure for a long time and serves as a reservoir for transmission of VRE to other patients (Patel, 2003). Within the genus Enterococcus, Enterococcus faecium has emerged as the most therapeutically challenging organism and the genomes indicate that there are certain genetic lineages which having ability to succeed in the hospital environment (Arias and Murray, 2012). Frequent colonization with VRE on medical intensive-care unit (MICU) and successive cross-colonization becomes the important factors in the endemic spread of VRE (Bonten et al., 1996); hence considering the spread of antibiotic- resistant enterococci and resistance genes, the emergence of VRE develops a non-existence barrier between the peoples and other animals also.

\section{Literature Review:-}

\section{Epidemiology of enterococcal infection:}

Enterococcus is referred as the "Streptococci of fecal origin". The term Enterococcus was used for the intestinal origin of these gram positive bacteria occurring in pairs and short chains which was published in a paper presentation in France in 1899.

The occurrence of Enterococcus spp. varies widely from animal, human to environmental sources. They form a part of microflora in human intestinal tract (E. faecalis and E. faecium); E. faecium is predominant in animals whereas $E$. mundtii and E. casseliflavus is observed in plants (Klein, 2003). The Enterococcus spp. are now becoming the second most causative agent of hospital infections (Collins et al., 1984). Montecalvo et al. (1995) reported that there was an increase in the number of patients colonized with VRE on screening the rectal swabs. According to them the main sites affected by VRE include urinary sites, intra-abdominal sites, surgical, blood stream and vascular sites.

E. faecalis and E. faecium are the two species predominantly associated with clinical infections. Collier and Sussman (2005) reported that E. faecalis accounts for around 80 to $90 \%$ followed by E. faecium that comprises of about $15 \%$ of the infections. Other species that can cause infections include E. avium, E. durans, E. gallinarum, $E$. casseiflavus etc. The infections caused by these species include urinary tract infections, endocarditis, intraabdominal and intra pelvic abscesses, blood stream infections and wound infections following surgery. Enterococci are found to be the most common cause of infections involving the urinary tract. Most of these infections have been reported in hospitalized individuals (Morrison et al., 1986). Apart from these Enterococci are also an important pathogens associated with intra-abdominal infections and sepsis. It also results in peritonitis in patients on long term dialysis (Gorensek et al., 1988). They have also been associated with salphingitis, maternal infections as endometritis and post Cesarean abscess formation (Gibbs et al., 1977). Bacteremia due to Enterococcus accounts to be the third most common cause of blood stream infections (Sood et al., 2008). Several studied report suggests that Enterococci accounts for nearly $15 \%$ of endocarditis. The main pathogen is E. faecalis but other species also play a role in this manifestation. Individuals above 65 years are more prone to Enterococcal endocarditis (Murray, 1990). The other risk factors involved are preceding UTI or catheterization, biliary infections. Group B streptococci and Escherichia coli are the main pathogens causing infections in neonates, but it has been recorded that Enterococcal species also may cause infections in this age groups. Infants with these infections had a remarkable feature of previous premature delivery, low birth weight and some cases long standing central line was recorded (Luginbuhl et al., 1987). Apart from neonates Enterococcal species can cause meningitis in older children and adults rarely. Most of them have a prior risk factor as an invasive procedure or long term infection and on prolonged antibiotic (Murray, 1990).

Hoffmann and Moellering (1987) reported that Enterococcal species to be the major cause of infections acquired from the hospitals. The recent studies proved that person - to - person transfer being the important source. It's been noted that treatment with antimicrobials without Enterococcal activity has led to superinfections. The use of polymyxin aerosol in the prevention of pneumonia associate with Pseudomonas has led to the spread of Enterococcal induced respiratory infections.

\section{Antibiotics and VRE detection and transmission:}

Besides being intrinsically resistant to various different classes of antibiotics, enterococci are able to acquired high level drug resistance through horizontal gene transfer. Cho et al., 2013 studied the impact of vancomycin resistance on mortality in neutropenic patients where they found that VRE bloodstream infection (BSI) is generally associated with the delayed administration of adequate antibiotics. Antibiotics can able to influence nosocomial VRE infections at different levels. In a patient with initially free of VRE, antibiotics might be able to decrease resistance against VRE colonization in the gastrointestinal tract and might be able to increase the susceptibility for colonized of 
microorganism (Harbarth et al., 2002). Recent molecular epidemiological studies have identified CC17, which is a globally isolated clonal lineage of E. faecium, characterized by resistance to ampicillin and quinolones (Leavis et al., 2006). The exposure of antibiotics also may increase the potential for transmission by causing stool in continence, which may enhance the risk of environmental contamination with VRE (Harbarth et al., 2002).

Schwartz et al. (2002) characterized VRE by antibiograms, and reported some resistance gene like vanA, Enterobacterial ampC resistance genes etc. and were detected by molecular biology methods and PCR from wastewater, surface water and drinking water biofilms. Volkmann et al. (2003) studied the antibiotic resistance genes vanA of enterococci in different municipal wastewater sample by using real-time PCR assay where the resistance genes were detected in $21 \%$ of samples. Edberg et al. (1994) studied the rapid detection methods of VRE and reported that the colonies grew on campylobacter blood agar with clindamycin medium were specifically identified as enterococci within $30 \mathrm{~min}$ via pyroglutamyl-I-naphthylamide or by rapid bile esculin tests.

Transmission of VRE by health care workers through hands is probably the most common mode of nosocomial transmission. Transmission of VRE may also occur by way of contaminated medical equipment, although this is probably much less of a factor than transmission by the hands of personnel (Weber et al., 1997). Tacconelli and Cataldo (2008) also support in this content. According to them transmission of VRE can occur through direct contact with colonized or infected patients or indirectly via hands of health care workers. And also the antibiotics exposure plays a vital role in transmission dynamic of VRE.

\section{Mechanism of vancomycin resistance:}

Out of five recognized phenotypes of vancomycin resistance among which VanA and VanB were described in Enterococcus spp. (Arthur and Courvalin, 1993). The third phenotype VanC was isolated E. gallinarum and E. casseliflavus and is characterized by moderate levels of vancomycin resistance. In compare with van $\mathrm{A}$ and vanB gene cluster, the genes drawn in the VanC phenotype (vanC1 and vanC2) are inherent and are naturally occurring genes (Murray et al., 1997). Usually Vancomycin and other glycopeptides inhibit the cell wall synthesis of bacteria by binding with terminal dipeptide, D-alanyl-D-alanine (D-ala-D-ala) of a pentapeptide containing cell wall precursor, which leads to prevent the precursor for participating further in the synthesis of cell wall. The basis for VRE is that the cell makes a precursor with a different terminus, primarily D-ala-D-lactate, to which vancomycin does not bind, and thus it does not inhibit cell wall synthesis of these bacteria (Murray et al., 1997). Arthur and Courvalin, (1993) documented vancomycin resistance gene by taking vanA gene cluster and it was found on the transposon, or “jumping” genetic element, Tn1546.

\section{Vancomycin dependent Enterococci:}

An interesting phenomenon that has developed in some strains of VanA and VanB type VRE is that of vancomycin dependence (Green et al., 1995; Dever et al., 1995). These enterococci are not only resistant to Vancomycin but require it for its growth. This group of Enterococci alters their normal production of D-Ala-D-Ala and is able to grow only in the presence of Vancomycin. This is due to the production of Van H (dehydrogenase) and ligase(Van A or Van B) that forms the D-Ala-D-Lac only if Vancomycin is present. When Vancomycin is not present then there is no D-Ala-D-Lac and without either of these Enterococci cannot survive. Reversion to Vancomycin independent form is observed which is due to mutation (Cetinkaya et al., 2000).

\section{Objectives:-}

To isolates and identify Enterococcus spp. collected from clinical, environmental samples and also to study the antibiotic susceptibility and determination of Vancomycin resistance pattern among all resistance isolates by MIC test.

\section{Methodology:-}

\section{Sample collection and transportation:}

Samples like urine, blood, stool etc are collected from Central referral hospital, Manipal and others are collected from sewage water for microbiological testing and are transported in sterile specimen containers or, in the case of fluid specimens syringes are used for transportation to the laboratory. 


\section{Isolation and identification of sample:}

Samples were first inoculated onto MacConkey agar, Bile esculin agar, M- Enterococcus agar, blood agar. The identification of the morphology was observed via gram staining method. MacConkey agar was used as a selective or differential medium for the cultivation of microorganism; here the samples were incubated at $37^{\circ} \mathrm{C}$ for 24 hours. Also Bile Esculin Agar (BEA) was done to identify enterococci and group D streptococci based on their ability to hydrolyze esculin, where bile esculin agar was taken in 500 $\mathrm{ml}$ distilled water and the media was autoclaved for 30 min., then on the prepared media samples were poured and incubated it at $37^{\circ} \mathrm{C}$. M-enterococcus agar was used for isolation and enumeration of Enterococcus spp. for sewage samples only, here the serial dilution methods was done for observing the inhibition of gram negative organisms. Also brain heart infusion (BHI) was done by adding 6.5\% $\mathrm{NaCl}$ for differentiation of enterococci from the collected sample. Blood agar media was used to grow fastidious organisms and differentiation of bacteria based on their hemolytic properties. For hemolysis test bacterial culture was inoculated onto the blood agar and was incubated at $37^{\circ} \mathrm{C}$ then the plate was observed after 24 hours.

\section{Physiological and biochemical test:}

Catalase test was done to identify the bacteria from the collected samples. Here a small amount of the test isolate is picked on a clean glass slide and $3 \% \mathrm{H}_{2} \mathrm{O}_{2}$ was added on it and mixed it with help of a loop to show the immediate production of gas bubbles from the surface of the glass slide. Coagulase test was also done for identification of species from the isolates. The test was done by placing a drop of physiological saline on each end of a slide then a portion of isolates was added and also a drop of human plasma was added on it. The clump of the organisms was observed immediately after $10 \mathrm{sec}$.

Methyl red (MR) test was done to distinguish between members of the family Enterobacteriaceae. It was prepared by dissolving $0.1 \mathrm{~g}$ of methyl red in $300 \mathrm{ml}$ of $95 \%$ ethyl alcohol. The organisms are taken from $18-24$ hour pure culture of the isolates and incubated it for 24 hours and then 2 to 3 drops of methyl red was added on it.

Voges proskauer test was done by preparation of MR-VP broth where $0.7 \mathrm{gm}$ peptone, $0.5 \mathrm{gm}$ of dextrose, and 0.5 gm potassium phosphate were added on $100 \mathrm{ml}$ distilled water solution. Then the MRVP broth test tubes were inoculated and incubated it at $37^{\circ} \mathrm{C}$ for $24-48$ hours. Then 5 drops of methy red indicator, 3-4 drops of alpha-napthol (Berrit reagent A), and potassium hydroxide (Berrit reagent $\mathrm{B}$ ) was added on it. The test was incubated till the reaction will occur.

Also the carbohydrate fermentation test was done to determine whether or not bacteria can ferment specific carbohydrates. Here the broth media was prepared by mixing all the ingredients (viz- Trypticase $10 \mathrm{~g}, \mathrm{NaCl} 5 \mathrm{~g}$, beef extract $1 \mathrm{~g}$, phenol red $0.018 \mathrm{~g}$, and carbohydrate source $10 \mathrm{~g}$ ) in $1000 \mathrm{ml}$ distilled water by applying heat. Then $4-5$ $\mathrm{ml}$ of broth was taken in each tube and inserted Durham tube to detect gas production. The prepared test media are sterilized and the $\mathrm{pH}$ was maintained it at $7.4 \pm 0.2$. The results were observed after 24 hours incubation.

Indole test was also done for differentiation of enterobacteriaceae. The indole test was performed by inoculating the test organism onto tryptophan broth and incubated it at $37^{\circ} \mathrm{C}$ for $24-48$ hours in ambient air. Then Indole was detected by adding kovac's reagent to the broth culture.

\section{Antimicrobial susceptibility test:}

All the 50 isolates were checked for the antimicrobial susceptibility pattern by using Kirby-Bauer disc diffusion method. Three or four pure colonies were inoculated in peptone water and incubated it for 3 hours. Then the turbidity of the growth was matched with Mc Farlands standards. A lawn culture of the broth with a sterile swab was

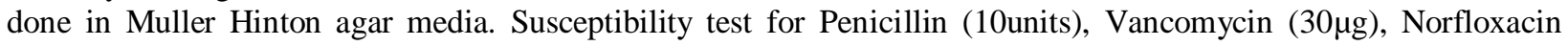
$(10 \mu \mathrm{g})$, Ampcillin $(10 \mu \mathrm{g})$, Streptomycin $(300 \mu \mathrm{g})$, High level gentamycin $(120 \mu \mathrm{g})$, Erytromycin $(15 \mu \mathrm{g})$ and Tetracycline $(30 \mu \mathrm{g})$ was done by following the National Committee for Clinical Laboratory guidelines (reference). Then the zones of inhibition were measured and recorded as per the recommendations from CLSI guidelines.

\section{Minimum Inhibitory test (MIC):}

Phenotypic characterization of Vancomycin resistance was based on the minimum inhibitory concentration (MIC) of the isolates to Vancomycin antibiotics by agar dilution methods. Muller hinton agar plates with antibiotics concentration in a range from $0.25 \mu \mathrm{g} \mathrm{ml}^{-1}$ (Tiwari et al., 2009) were prepared. For preparing the gradient first a stock concentration of $500 \mu \mathrm{g} \mathrm{ml}^{-1}$ was prepared from where different antibiotic concentration were prepared by using following formula - 
$\mathrm{V}_{1} \mathrm{~S}_{1}=\mathrm{V}_{2} \mathrm{~S}_{2}$ (where $\mathrm{V}_{1}=$ Volume of the stock solution, $\mathrm{S}_{1}=$ Concentration of the stock solution, $\mathrm{V}_{2}=$ Final volume of the solution, $S_{2}=$ Concentration of solution to be prepared)

The medium is poured in sterile Petri plates and allowed to solidify. The plates were marked according to their antibiotic concentration and the test samples were prepared by mixing 1 or 2 colonies from actively grown culture into sterile $0.8 \% \mathrm{NaCl}$ solution and compared it with McFarland Standard 0.5. The plates were then incubated at $37^{\circ} \mathrm{C}$ for 24 hours. After the incubation the plates are checked for visible growth at the time inoculation sites

\section{Results:-}

\section{Results of identification test:}

All the 50 isolates collected from various clinical and environmental sources including urine, poultry farm, sewage were processed for gram staining and the results shows that all the isolates were gram positive cocci with a pairs of short chain like appearance (fig.1). In the MacConkey agar medium tiny lactose fermenting colonies were observed (fig.2). The results of Bile Esculin Agar (BEA) test indicates that the most of the isolates can able to hydrolyze esculin in presence of bile (fig.3). In M-Enterococcus agar media plates dark or light red color round colonies were observed (fig.4), also test report from BHI broth showed the positive results amongst isolates, it can able to tolerate high salt content $(6.5 \% \mathrm{NaCl}$ in $\mathrm{BHI}$ broth). So, from this observation it confirmly indicates that the collected isolates mostly contains Enterococci spp.

\section{Results of physiological and biochemical test:}

The catalase test showed negative result because there was not any bubble formation occurs on the slide in presence of $3 \% \mathrm{H}_{2} \mathrm{O}_{2}$ whereas Staphylococcus aureus showed positive results which was taken as a positive control. The coagulase test also showed negative results, as there was not any clot formation appeared on the plasma of the slides.

Results of blood agar hemolysis for Enterococcus spp. showed variability for alpha, beta, and gamma hemolysis (Fig. 5). Enterococcus is the catalase negative organism, and hence there was no bubbles formation on the glass slides, where Staphylococcus aureus was taken as positive control. In Coagulase test for Enterococcus spp. showed negative result, also in MR-VP test Enterococcus shows methyl negative, as there was no color change in presence of methyl red indicator, but in case of Voges proskauer test Enterococcus spp. showed the positive results due to presence of alpha-naphthol and potassium (Fig. 6).

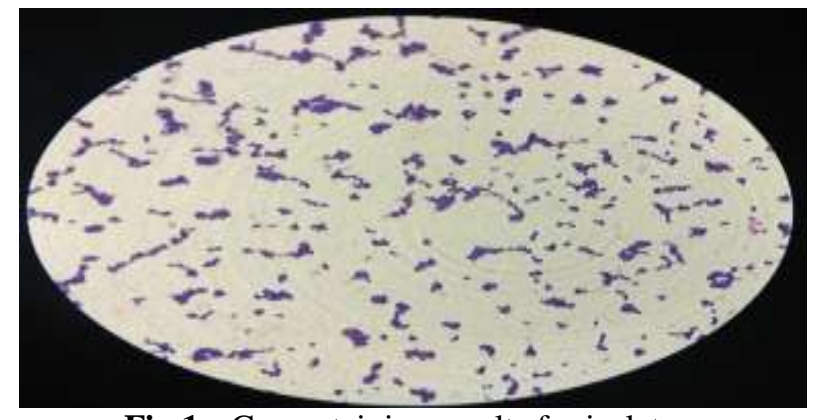

Fig 1:- Gram staining results for isolates.

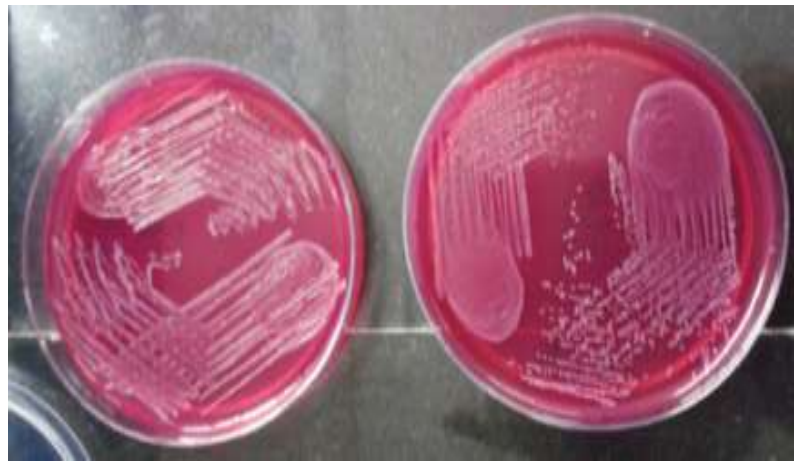

Fig 2:- MacConkey agar plate showing lactose fermenting colonies. 


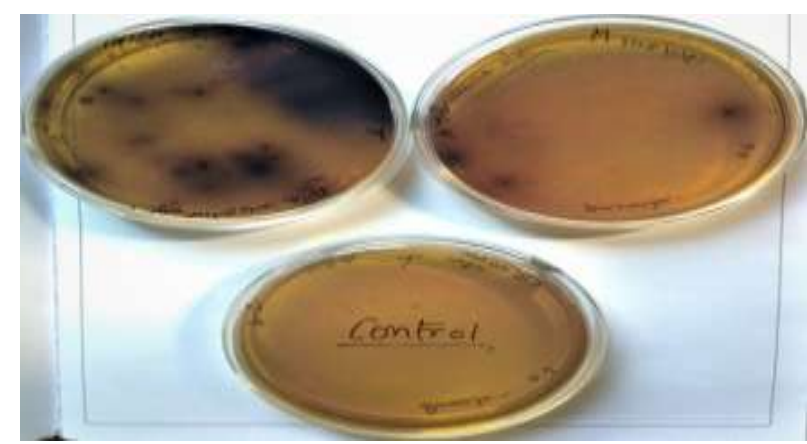

Fig 3:- Bile esculin agar plates showing esculin in presence of bile.

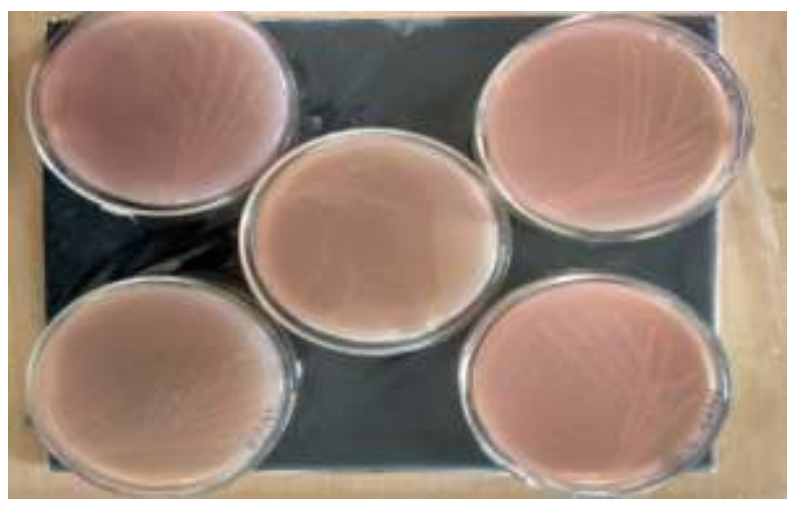

Fig 4:- M-enterococcus selective agar plates.

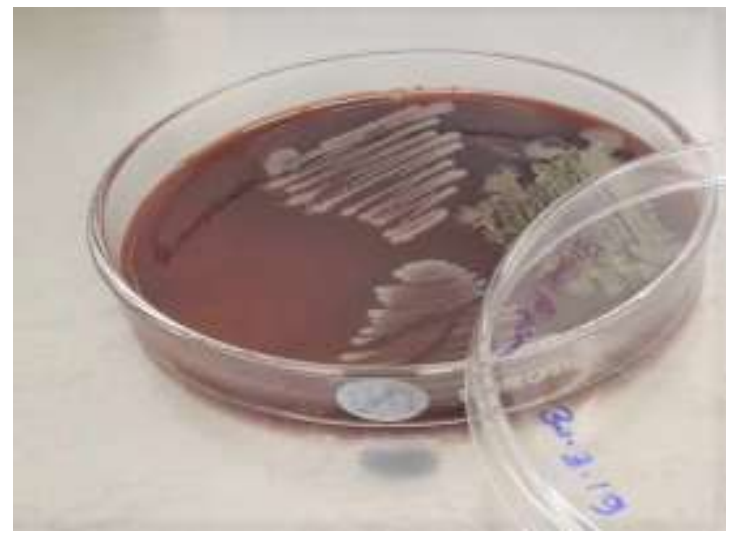

Fig 5:- Blood agar plate of Enterococcus spp.

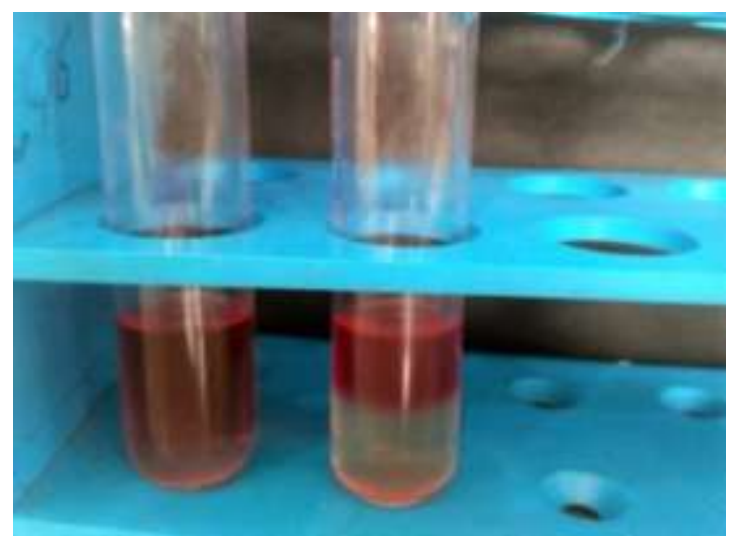

Fig 6:- Voges proskauer test of Enterococcus spp. 
Standard biochemical test depicts the distribution of enterococcal species among the various samples. E. faecalis shows positive results to mannitol, glucose, sorbitol, melibiose (Fig. 7, 8, 9, and 10 respectively); and negative results to arabinose, arbutin. Results of Indole test clearly showed that there was not any appearance of red/pink color ring like layer on the surface of the broth. Hence Enterococcus spp was indole negative organism.

\section{Results of antibiotics susceptibility and MIC test:}

Kirby Bauer's disc diffusion test was done for antibiotic susceptibility test. Out of 50 isolates ampicillin sensitive strain were 40 , and resistant strain were 10 , HLG sensitive strains were $45 \&$ resistant strain were 5, Erythromycin sensitivity were observed in 39 isolates and resistant in 11 isolates, vancomycin sensitive strain were 18 and resistant were 35 isolates, Norfloxacin sensitive strain were $33 \&$ resistant strain were 17 strain, Penicillin sensitive strain were 35 and resistant strain were 15 , Tetracycline sensitive strain were 33 and resistant strain were 17, Streptomycin sensitive strain were 41 and resistant were 9 . The maximum sensitivity was observed followed by ampicillin and high level gentamicin (HLG) and streptomycin. MIC test was done on (35) VRE strain, out of which (10) strains showed high resistance at higher concentration $(0.5 \mu \mathrm{g} / \mathrm{ml}-32 \mu \mathrm{g} / \mathrm{ml})$.

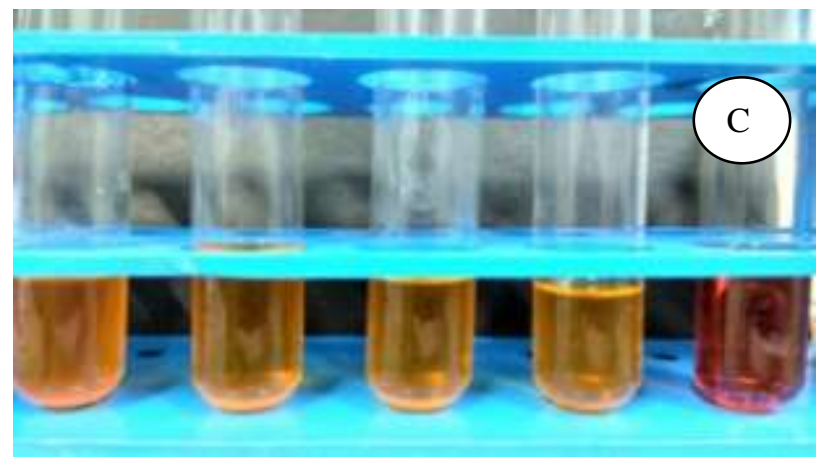

Fig.7:- Sugar fermentation test of mannitol showing positive results to enterococci, $\mathrm{C}=$ control.

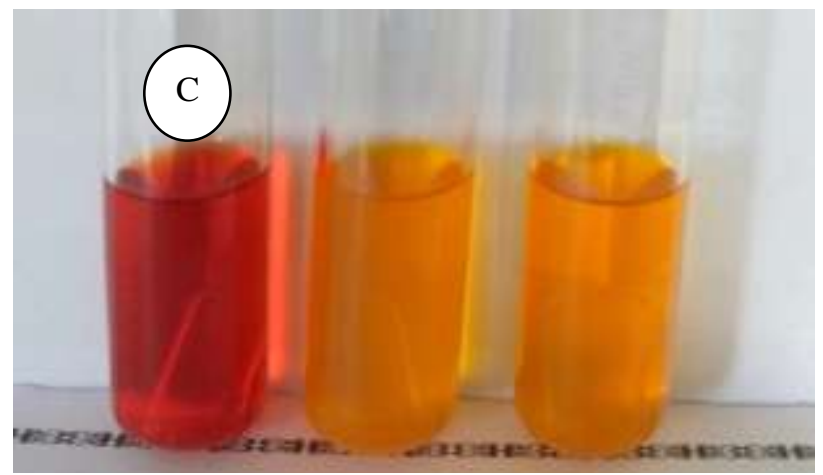

Fig. 8:- Sugar fermentation test of glucose showing positive results to enterococci, $\mathrm{C}=$ control.

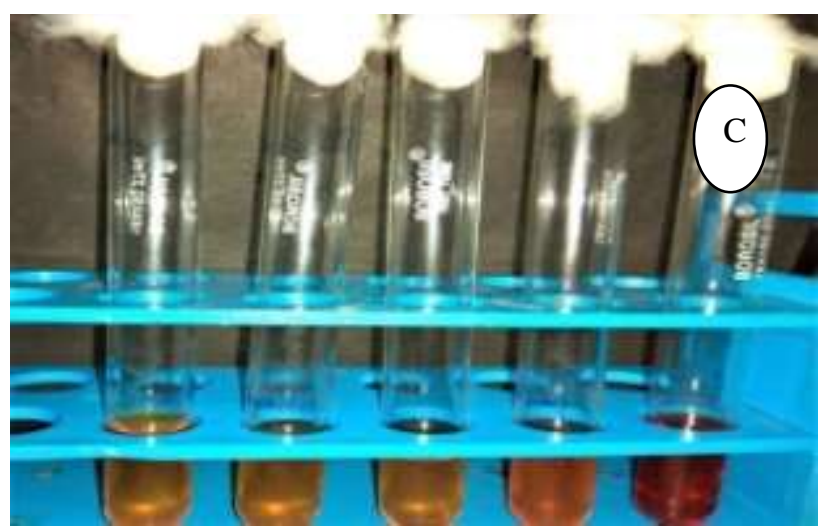

Fig. 9:- Sugar fermentation test of sorbitol showing positive results to enterococci, $\mathrm{C}=$ control. 


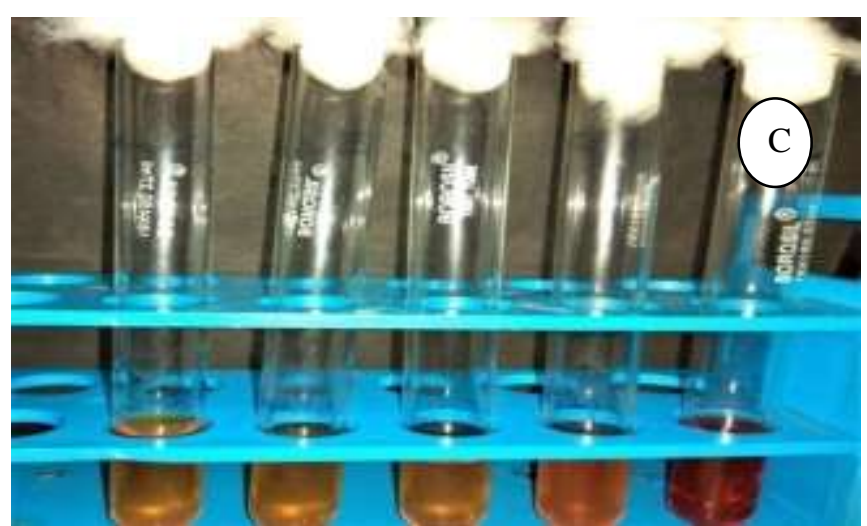

Fig. 10:- Sugar fermentation test of melibios shows positive results to enterococci, $\mathrm{C}=$ control.

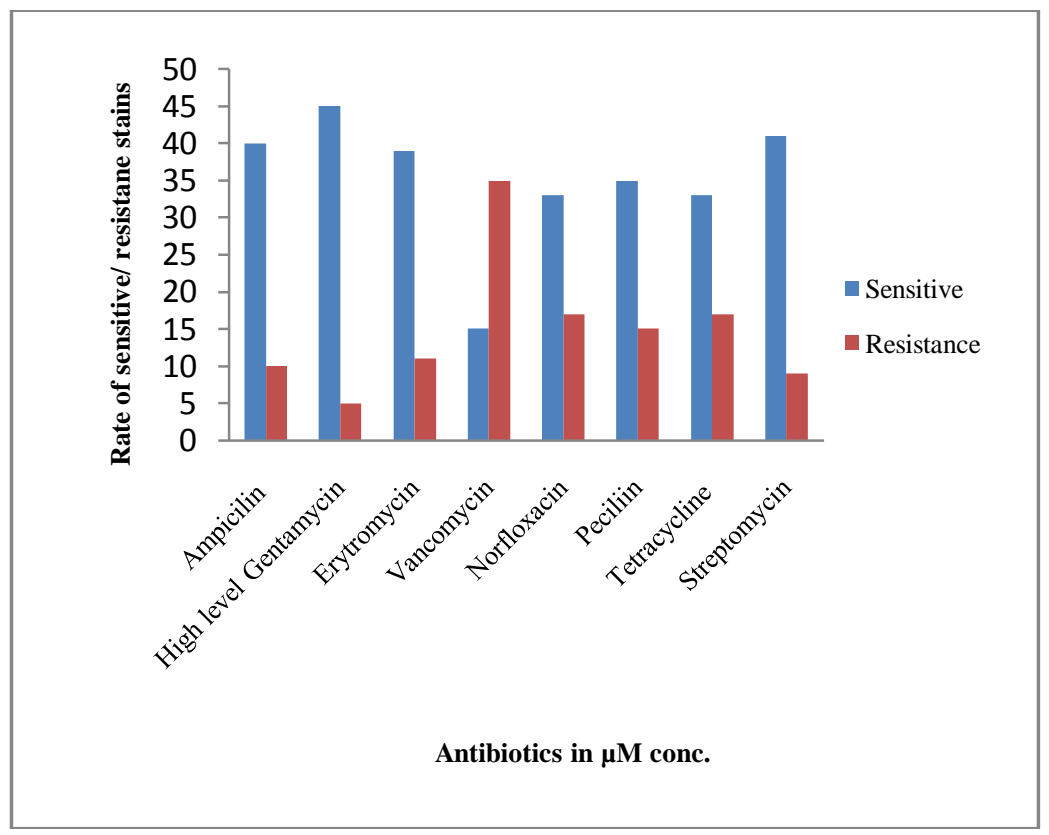

Fig. 11:- Treatment of different antibiotics to Enterococcus isolated for detection of resistivity.

\section{Discussion and Conclusion:-}

Enterococcal infections are becoming one of the most important health problems which can causes considerable morbidity in human population. In this study the microbiologic tests was applied to aid in the identification of an unknown culture, because it is necessary to have some knowledge of the culture's gram staining characteristic, cellular arrangement, catalase and hemolytic activity. This was the case for the presumptive tests described here.

All the 50 isolates of Enterococcus from various clinical and environmental samples including urine, sewage, and poultry farm were gram positive and coccal shaped. In our finding it was seen that Enterococcus spp. are catalase negative and grew in the presence of bile salts. Hence all the isolates that satisfied this preliminary identification criterion were subjected to further confirmatory tests.

Carbohydrate fermentation tests were conducted which shows different fermentation characteristic with respect to carbohydrates. Yun et al., (2003) also reported that when E. faecalis RKY1 were grown on fermentation medium containing glucose, fructose and maltose; they produce lactic acid with a high yield via homofermentative pathway.

Banerjee and Anupurba (2015) studied the two major species associated with most of the enterococcal infections, viz, E. faecalis and E. faecium where they found that E. faecalis the commonly reported species and are the major isolated species in many centres. They reported that with increasing the drug resistance in enterococci becomes 
responsible for the emergence of E. faecium as a dominant species especially from VRE isolates. So, this result is in agreement with our findings because these species are intrinsically resistance to the number of antibiotics and having the capability to endure in harsh environment. One of the most important characteristic for the evaluation of enterococci is their susceptibility for different antibiotics. Ateba and Maribeng (2011) also isolated three different species of enterococci species were E. faecium was found to be the most predominant in the entire studied site. They also reported that the enterococci species were resistant to sulphamethothazole, amoxicillin, erythromycin,

Table 1:- MIC test results of different concentration of vancomycin antibiotics; here '+' sign indicates the resistivity to desired conc.

\begin{tabular}{|c|c|c|c|c|c|c|c|}
\hline No. of isolates & \multicolumn{7}{|c|}{ Desire conc. in $\mu \mathrm{g} \mathrm{ml}^{-1}$ (range of inhibition) } \\
\hline & 0.5 & 1 & 2 & 4 & 8 & 16 & 32 \\
\hline 1 & + & + & & & & & \\
\hline 2 & + & + & + & & & & \\
\hline 3 & + & + & + & & & & \\
\hline 4 & + & + & + & + & + & & \\
\hline 5 & + & + & + & & & & \\
\hline 6 & + & + & + & + & + & + & \\
\hline 7 & + & + & + & & & & \\
\hline 8 & + & + & + & & & & \\
\hline 9 & + & + & + & + & & & \\
\hline 10 & + & + & + & + & + & & \\
\hline 11 & + & + & + & & 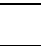 & & \\
\hline 12 & + & + & + & + & + & + & + \\
\hline 13 & + & + & + & + & & & \\
\hline 14 & + & + & + & + & + & & \\
\hline 15 & + & + & + & + & & & \\
\hline 16 & + & + & + & + & + & + & + \\
\hline 17 & + & + & + & + & + & & \\
\hline 18 & + & + & + & + & + & + & + \\
\hline 19 & + & + & + & + & & & \\
\hline 20 & + & + & + & + & + & + & \\
\hline 21 & + & + & + & + & + & & \\
\hline 22 & + & + & + & + & + & + & + \\
\hline 23 & + & + & + & + & + & & \\
\hline 24 & + & + & + & + & + & + & \\
\hline 25 & + & + & + & + & + & & \\
\hline 26 & + & + & + & + & + & + & + \\
\hline 27 & + & + & + & + & + & + & + \\
\hline 28 & + & + & + & + & + & + & + \\
\hline 29 & + & + & + & + & + & & \\
\hline 30 & + & + & + & + & & & \\
\hline 31 & + & + & + & + & + & + & \\
\hline 32 & + & + & + & + & + & + & + \\
\hline 33 & + & + & + & + & + & & \\
\hline 34 & + & + & + & + & + & + & + \\
\hline 35 & + & + & + & + & + & + & + \\
\hline
\end{tabular}

Chloramphenicol, vancomycin, tetracycline and penicillin. Majhenic et al., (2003) reported that enterococci was very resistance to clindamycin only, vancomycin susceptibility test was analysed with E- test, where they revealed that the strain was sensitive to vancomycin. Simjee et al., (2007) also described that the isolates recovered from poultry litter, where 90 enterococcus isolates were tested and 96\% among them were often resistance to tetracylin, followed by clindamycin (90\%). Yurdakul et al., (2013) studied on antibiotic resistance of Enterococci where they found that $100 \%$ of the isolate which was collected from chicken meat were resistant to tetracycline, $75 \%$ of them were resistance to ciprofloxacin, and $50 \%$ of them were resistance to erythromycin, Vancomycin and 
chloramphenicol. So, this result is not in agreement with our finding. In our study Vancomycin resistivity was found to be the highest (70\%) then other antibiotics, also MIC test report revealed that out of $70 \%$ of VRE strain $29 \%$ of strains showed highest resistance at higher concentration $(0.5 \mu \mathrm{g} / \mathrm{ml}-32 \mu \mathrm{g} / \mathrm{ml})$. The role of enterococci in the transfer of antibiotic resistance traits is still under debate and increasing interest in the epidemiology of these bacteria continues worldwide.

Considerable commonness of pathogenic prominent species of enterococci was seen along with their emerging drug resistance and virulence. Complete identification and routine speciation is necessary to limit their appearance as major species in near future. From the above study, we concluded that in enterococcal isolates, virulence resolute have been extensively common was taken from clinical origin, on the other hand they were exist complex and a strict regulation mechanism of expression for this, pathogenecity determinants especially with respect to antibiotic resistance in enterococci. This study summarizes the experiential association of virulence and drug resistance in enterococci and enunciates the need for more research on the role of horizontal gene transfer and defence mechanisms of enterococci.

\section{Acknowledgement:-}

We thank all the faculty members and technical staff of School of Life Science, Sikkim University for their encouragement and supports; also thanks to Central Referral Hospital (Manipal, Gangtok) for technical assistance and support in the collection of specimens from hospital to carry out the entire project.

\section{Reference:-}

1. Arias, C. A., \& Murray, B. E. (2012). The rise of the Enterococcus: beyond vancomycin resistance. Nature reviews microbiology, 10(4), 266-278.

2. Arthur, M., \& Courvalin, P. (1993). Genetics and mechanisms of glycopeptide resistance in enterococci. Antimicrobial agents and chemotherapy, 37(8), 1563.

3. Ateba, C. N., \& Maribeng, M. D. (2011). Detection of Enterococcus species in groundwater from some rural communities in the Mmabatho area, South Africa: A risk analysis. African journal of microbiological research, 5(17), 3930-3935.

4. Banerjee, T., \& Anupurba, S. (2015). Prevalence of virulence factors and drug resistance in clinical isolates of Enterococci: A study from North India. Journal of pathogens, 2015.

5. Bonten, M. J., Hayden, M. K., Nathan, C., van Voorhis, J., Matushek, M., Slaughter, S., ... \& Weinstein, R. A. (1996). Epidemiology of colonisation of patients and environment with vancomycin-resistant enterococci. The lancet, 348(9042), 1615-1619.

6. Bonten, M. J., Willems, R., \& Weinstein, R. A. (2001). Vancomycin-resistant enterococci: why are they here, and where do they come from?. The lancet infectious diseases, 1(5), 314-325.

7. Cetinkaya, Y., Falk, P., \& Mayhall, C. G. (2000). Vancomycin-resistant enterococci. Clinical microbiology reviews, 13(4), 686-707.

8. Cho, S. Y., Lee, D. G., Choi, S. M., Kwon, J. C., Kim, S. H., Choi, J. K., ... \& Yoo, J. H. (2013). Impact of vancomycin resistance on mortality in neutropenic patients with enterococcal bloodstream infection: a retrospective study. BMC infectious diseases, 13(1), 504.

9. Collier, L. B., \& A Sussman, M. (1998). Topley \& Wilson's Microbiology and microbial infections. Bacterial infections, 3.

10. Collins, M. D., Jones, D., Farrow, J. A. E., Kilpper-Balz, R., \& Schleifer, K. H. (1984). Enterococcus avium nom. rev., comb. nov.; E. casseliflavus nom. rev., comb. nov.; E. durans nom. rev., comb. nov.; E. gallinarum comb. nov.; and E. malodoratus sp. nov. International journal of systematic and evolutionary microbiology, 34(2), 220-223.

11. Dever, L. L., Smith, S. M., Handwerger, S., \& Eng, R. H. (1995). Vancomycin-dependent Enterococcus faecium isolated from stool following oral vancomycin therapy. Journal of clinical microbiology, 33(10), 27702773.

12. Edberg, S. C., Hardalo, C. J., Kontnick, C., \& Campbell, S. (1994). Rapid detection of vancomycin-resistant enterococci. Journal of clinical microbiology, 32(9), 2182-2184.

13. Gibbs, R. S., Listwa, H. M., \& Dreskin, R. B. (1977). A pure Enterococcal abscess after cesarean section. The Journal of reproductive medicine, 19(1), 17-20.

14. Gorensek, M. J., Lebel, M. H., \& Nelson, J. D. (1988). Peritonitis in children with nephrotic syndrome. Pediatrics, 81(6), 849-856. 
15. Green, M., Shlaes, J. H., Barbadora, K., \& Shlaes, D. M. (1995). Bacteremia due to vancomycin-dependent Enterococcus faecium. Clinical infectious diseases, 20(3), 712-714.

16. Harbarth, S., Cosgrove, S., \& Carmeli, Y. (2002). Effects of antibiotics on nosocomial epidemiology of vancomycin-resistant enterococci. Antimicrobial agents and chemotherapy, 46(6), 1619-1628.

17. Hoffmann, S. A., \& Moellering, R. C. (1987). The Enterococcus: Putting the bug in our ears. Annals of internal medicine, 106(5), 757-761.

18. Kak, V., \& Chow, J. W. (2002). Acquired antibiotic resistances in enterococci. American society of microbiology, 355-383.

19. Klein, G. (2003). Taxonomy, ecology and antibiotic resistance of enterococci from food and the gastrointestinal tract. International journal of food microbiology, 88(2-3), 123-131.

20. Leavis, H. L., Bonten, M. J., \& Willems, R. J. (2006). Identification of high-risk enterococcal clonal complexes: global dispersion and antibiotic resistance. Current opinion in microbiology, 9(5), 454-460.

21. Luginbuhl, L. M., Rotbart, H. A., Facklam, R. R., Roe, M. H., \& Elliot, J. A. (1987). Neonatal Enterococcal sepsis: case-control study and description of an outbreak. The pediatric infectious disease journal, 6(11), 10221026.

22. Majhenič, A. Č., Rogelj, I., \& Perko, B. (2005). Enterococci from Tolminc cheese: population structure, antibiotic susceptibility and incidence of virulence determinants. International journal of food microbiology, 102(2), 239-244.

23. Montecalvo, M. A., de Lencastre, H., Carraher, M., Gedris, C., Chung, M., VanHorn, K., \& Wormser, G. P. (1995). Natural history of colonization with vancomycin-resistant Enterococcus faecium. Infection control \& hospital epidemiology, 16(12), 680-685.

24. Moore, W. E. C., \& Holdeman, L. V. (1974). Human fecal flora: the normal flora of 20 JapaneseHawaiians. Applied and environmental microbiology. 27(5), 961-979.

25. Morrison, A. J., \& Wenzel, R. P. (1986). Nosocomial urinary tract infections due to Enterococcus: ten years' experience at a university hospital. Archives of internal medicine, 146(8), 1549-1551.

26. Murray, B. E. (1997). Vancomycin-resistant enterococci. The American journal of medicine, 102(3), 284-293.

27. Murray, B. E. (1990). The life and times of the Enterococcus. Clinical microbiology reviews, 3(1), 46-65.

28. Mutters, N. T., Brooke, R. J., Frank, U., \& Heeg, K. (2013). Low risk of apparent transmission of vancomycinresistant Enterococci from bacteraemic patients to hospitalized contacts. American journal of infection control, 41(9), 778-781.

29. Patel, R. (2003). Clinical impact of vancomycin-resistant enterococci. Journal of antimicrobial chemotherapy, 51(suppl_3), iii13-iii21.

30. Schwartz, T., Kohnen, W., Jansen, B., \& Obst, U. (2003). Detection of antibiotic-resistant bacteria and their resistance genes in wastewater, surface water, and drinking water biofilms. FEMS microbiology ecology, 43(3), 325-335.

31. Simjee, S., McDermott, P. F., White, D. G., Hofacre, C., Berghaus, R. D., Carter, P. J., ... \& Maurer, J. J. (2007). Antimicrobial susceptibility and distribution of antimicrobial-resistance genes among Enterococcus and coagulase-negative Staphylococcus isolates recovered from poultry litter. Avian diseases, 51(4), 884-892.

32. Sood, S., Malhotra, M., Das, B. K., \& Kapil, A. (2008). Enterococcal infections \& antimicrobial resistance. Indian journal of medical research, 128(2), 111.

33. Tacconelli, E., \& Cataldo, M. A. (2008). Vancomycin-resistant enterococci (VRE): transmission and control. International journal of antimicrobial agents, 31(2), 99-106.

34. Volkmann, H., Schwartz, T., Bischoff, P., Kirchen, S., \& Obst, U. (2004). Detection of clinically relevant antibiotic-resistance genes in municipal wastewater using real-time PCR (TaqMan). Journal of microbiological methods, 56(2), 277-286.

35. Weber, D. J., \& Rutala, W. A. (1997). Role of environmental contamination in the transmission of vancomycinresistant enterococci. Infection control \& hospital epidemiology, 18(5), 306-309.

36. Yun, J. S., Wee, Y. J., \& Ryu, H. W. (2003). Production of optically pure L (+)-lactic acid from various carbohydrates by batch fermentation of Enterococcus faecalis RKY1. Enzyme and microbial technology, 33(4), 416-423.

37. Yurdakul, N. E., ErgiNkaYa, Z., \& ÜNal, E. (2013). Antibiotic resistance of Enterococci, coagulase negative Staphylococci and Staphylococcus aureus isolated from chicken meat. Czech journal of food sciences, 31(1), 14-19.

38. Zirakzadeh, A., \& Patel, R. (2006). Vancomycin-resistant Enterococci: colonization, infection, detection, and treatment. Mayo clinic proceedings, 81(4), 529-536. 